\title{
Man With Spontaneous Intracranial Hemorrhage on Therapeutic ENoxaparin, Clopidogrel, and Aspirin
}

\author{
Mihir K. Patel, MD, Sumeet K. Chhabra, MD, Michael Pfeiffer, MD
}

\section{Case Presentation}

A 65 year-old Caucasian male originally presented to an outside hospital complaining of worsening paroxysmal nocturnal dyspnea, orthopnea, and recent exertional chest pain associated with dyspnea. The patient's past medical history was significant for coronary artery disease status post coronary bypass, severe aortic stenosis status post bioprosthetic aortic valve repair, congestive heart failure, atrial fibrillation on anticoagulation, dual-chamber pacemaker placement, history of a transient ischemic attack, and type 2 diabetes mellitus. At the outside hospital, coronary angiography revealed occlusion of native vessels and previous grafts. He was considered a poor surgical candidate. He was transferred to Thomas Jefferson University Hospital (TJUH) for a second opinion regarding percutaneous versus surgical intervention.

The patient's initial hospital course was complicated by persistent volume overload treated with aggressive intravenous (IV) diuresis and atrial fibrillation refractory to medical therapy. Electrophysiologyservice recommended elective transesophageal echocardiogram (TEE) and electrocardioversion; TEE revealed no left atrial clot, and the procedure was successful in restoring sinus rhythm. Throughout his hospitalization, the patient was maintained on weight-adjusted therapeutic low molecular weight heparin (LMWH, enoxaparin), clopidogrel, and aspirin. Other medications included amiodarone, atorvastatin, digoxin, esomeprazole, ezetimibe, isosorbide mononitrate, metoprolol succinate (XL), spironolactone, levothyroxine, niacin, repaglinide, sertraline, and insulin.

Approximately two weeks after transfer to TJUH and two days after cardioversion, the housestaff was called overnight for an acute change in mental status and blurry vision. Upon evaluation, patient was unable to respond to questions appropriately. He complained of "not feeling well" and reported new bilateral blurry vision. The patient and nursing staff denied any recent trauma or fall. He was afebrile, normotensive (130/70) with normal sinus rhythm $(64 \mathrm{bpm})$, saturating well $(98 \%)$ in no respiratory distress (18 resps/min) with finger-stick blood glucose of $93 \mathrm{mg} / \mathrm{dL}$. Exam revealed the patient was oriented only to person; Glasgow Coma Scale (GCS) on initial evaluation was 14. Complete neurologic exam was non-focal: pupils were equal in size and reactive to light and accommodation; visual fields were intact bilaterally; cranial nerves II-XII were intact and symmetrical; muscle strength was full in his upper and lower extremities bilaterally; and his distal upper and lower extremity sensation was intact and symmetric.

Stat head CT (Figure 1) revealed a large intraparenchymal hemorrhage in the left parietal and temporal lobes, involving the left lateral ventricle, third ventricle, and partial filling of right

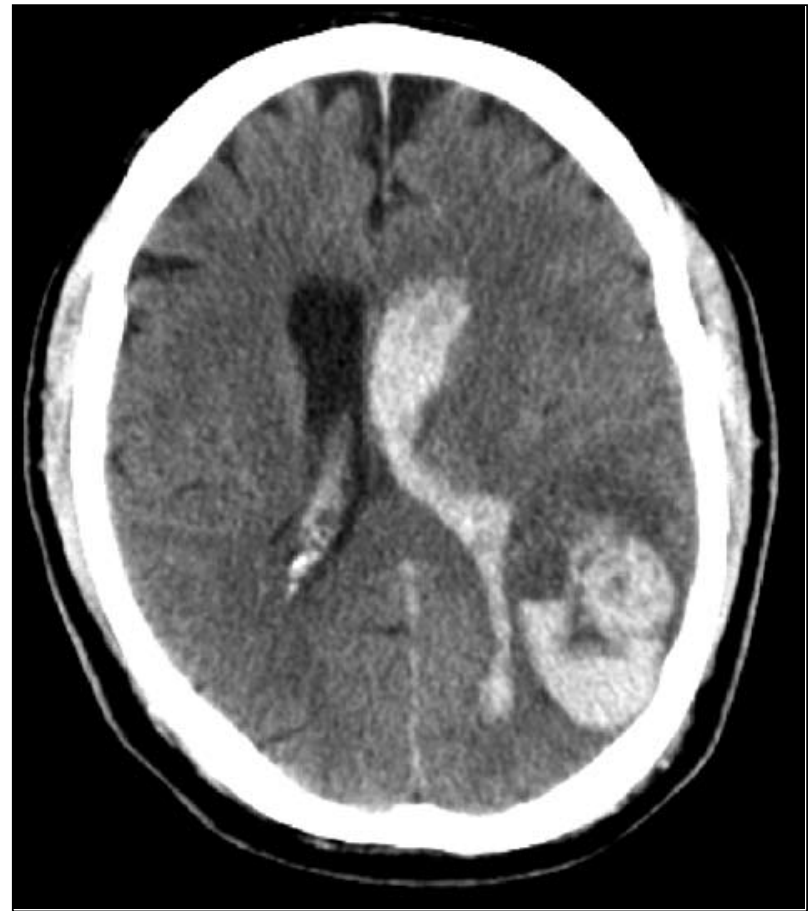

Figure 1.

Head CT without contrast shows a large intraparenchymal hemorrhage in the left parietal and temporal lobes with extension into the ventricles. Associated $7 \mathrm{~mm}$ left-to-right midline shift with local mass effect is also observed.

lateral and fourth ventricles. A $7 \mathrm{~mm}$ left-to-right midline shift with local mass effect in the left parietal lobe was also observed. Repeat neurological exam immediately after report of these findings remained non-focal, but the patient was notably more lethargic. His eyes closed spontaneously but opened to voice, and he responded to questions slowly and sometimes required repeated questioning; GCS at this time was 13 .

Neurosurgery evaluated the patient and arranged transfer to the Neurological ICU for emergent right frontal ventriculostomy. Per consultation with hematology, the patient received enoxaparin-adjusted IV protamine sulfate to attempt reversal of therapeutic enoxaparin. The patient's clopidogrel and aspirin were also discontinued. The patient remained normotensive throughout these events; however, his mental status and level of consciousness continued to deteriorate rapidly. Despite additional IV protamine sulfate, fresh frozen plasma, and factor IX, the hematoma continued to expand as demonstrated by repeat head CT (Figure 2) performed 10 hours after the initial diagnosis. Further surgical options were discussed with the 


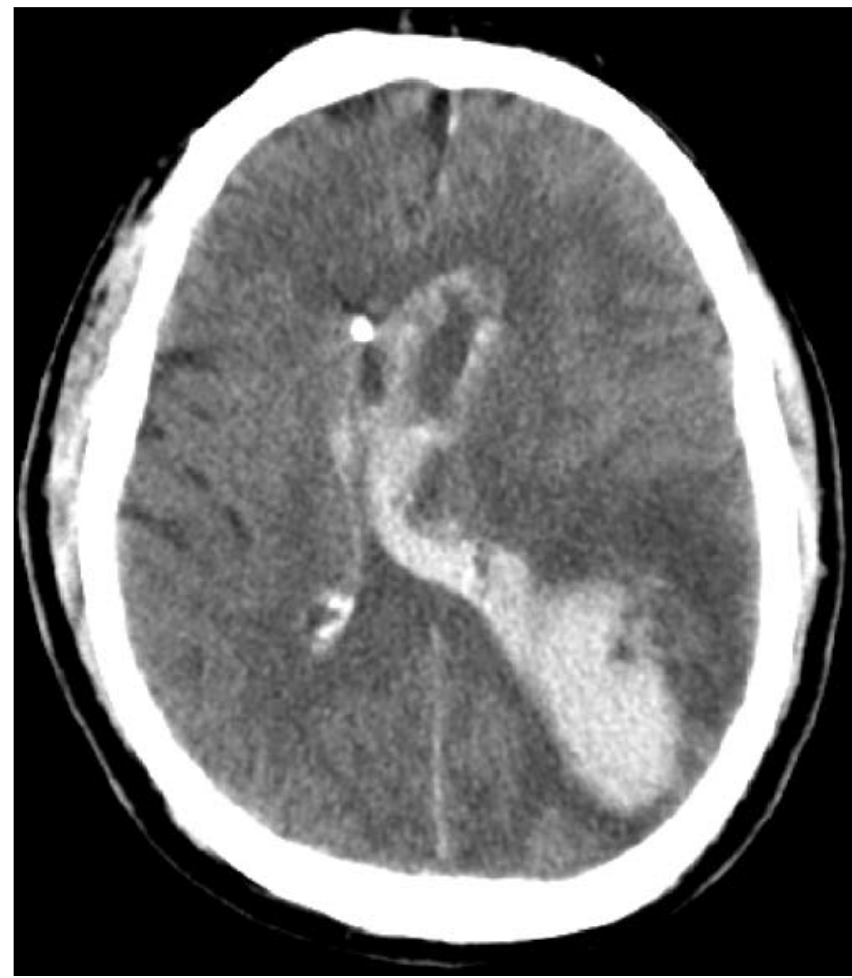

Figure 2

On a repeat head CT performed ten hours later, there is significant expansion of the left intraparenchymal hematoma and ventricular hemorrhage, with increasing rightward midline shift. Interval placement of a right frontal ventriculostomy catheter is noted.

patient's wife. Considering the low likelihood of significant functional recovery and her knowledge of the patient's wishes, surgical options were declined. He was subsequently placed on a morphine infusion for comfort and expired the next evening.

\section{Discussion}

According to the American Stroke Association, intracerebral hemorrhages (ICH) affected an estimated 67,000 individuals in 2002 , and only $20 \%$ of those individuals were expected to be functionally independent six months after the event. The 30 -day mortality of an ICH is reported to be 35 to $52 \%$, with the majority of deaths occurring within the first 2 days. Rapid recognition is critical due to the potential for quick deterioration of patients with this condition. The classic presentation is the sudden onset of a focal neurological deficit that worsens over minutes to hours. Other symptoms and signs include headache, vomiting, and decreased level of consciousness. There is usually a smooth progression of the neurological deficit over time. This progression is uncommon in ischemic strokes and subarachnoid hemorrhages. ${ }^{1}$ Nevertheless, clinical features alone are not sufficient to differentiate between ischemic and hemorrhagic strokes, and therefore, imaging is crucial. CT and MRI appear to be equal in detecting an $\mathrm{ICH}$, as well as determining size, location, and extent of hematoma enlargement. Generally, CT is superior in detecting ventricular extension, while MRI is superior in detecting structural lesions, amount of surrounding edema, and herniation. ${ }^{1}$ Because of the urgency associated with a concern for ICH combined with the availability and duration of MRI scans, CT is more commonly obtained.

Other aspects of the initial evaluation include obtaining vital signs, laboratory studies, an EKG, and a chest x-ray. In a study conducted by the Spanish Neurological Society, a temperature greater than $37.5 \mathrm{C}^{\circ}$, an elevated serum neutrophil count, and an elevated fibrinogen level were all associated with early neurological deterioration. ${ }^{2}$

Patients diagnosed with an ICH need ICU level care along with neurosurgical evaluation or transfer to a facility with neurosurgical capabilities. The major aspects of medical management for ICH involve controlling blood pressure, decreasing intracranial pressure, and treating associated conditions, such as hyperthermia, concomitant infections, or hyperglycemia. Any anticoagulation should be immediately discontinued or reversed if possible. Treating fevers with antipyretics is valuable because lower body temperature lessens tissue damage by redistributing oxygen and decreases glucose consumption to permit relative tolerance to oxygen deprivation. The data for treating hyperglycemia has been extrapolated from the data on ischemic strokes. Hyperglycemia in the first 24 hours ( $>140 \mathrm{mg} / \mathrm{dL}$ ) after an ICH is associated with worse outcomes, and current guidelines recommend the use of insulin for blood glucose levels over 140 to 185 . Ongoing research should provide more specific information and clarify these guidelines. ${ }^{1}$

Treatment of elevated intracranial pressures should start with simple measures such as elevating the head of the bed to thirty degrees and keeping the head in midline position. If more aggressive measures are needed, options include IV mannitol to achieve plasma osmolality of 300 to $310 \mathrm{mosmol} / \mathrm{kg}$, barbiturate coma, or hyperventilation to a $\mathrm{P}_{\mathrm{a}} \mathrm{CO}_{2}$ of 25 to $30 \mathrm{~mm} \mathrm{Hg}$. These patients also need concomitant measuring of intracranial pressure and blood pressure. ${ }^{1}$ Steroids should not be used as they increase complication rates, particularly infection, and have not been shown to improve outcomes. ${ }^{3}$ Many clinicians also use antiepileptic agents for seizure prophylaxis. A study in Italy aimed at characterizing ICH-related seizure revealed that use of antiepileptic agents soon after ICH onset could reduce the risk of early seizures. ${ }^{4}$ However, this benefit has yet to be substantiated by prospective clinical studies.

There are no clear guidelines on how to manage elevated blood pressure in ICH. On one hand, lowering the blood pressure potentially slows hematoma expansion. On the other hand, lowering blood pressure can also induce cerebral ischemia in the edematous portions surrounding the hemorrhage. Current 
guidelines, as outlined in Table 1, recommend using continuous IV infusion of antihypertensive agents for SBP > 200 or MAP $>150 \mathrm{~mm} \mathrm{Hg}$, and either continuous or intermittent IV medications for SBP > 180 or MAP > $130 \mathrm{~mm} \mathrm{Hg}$. Ongoing trials are specifically evaluating the control of blood pressure in patients with $\mathrm{ICH}{ }^{1}$

Table 1. Suggested Recommended Guidelines for Treating Elevated Blood Pressure in Spontaneous ICH

1. If $\mathrm{SBP}$ is $>200 \mathrm{~mm} \mathrm{Hg}$ or MAP is $>150 \mathrm{~mm} \mathrm{Hg}$, then consider aggressive reduction of blood pressure with continuous intravenous infusion, with frequent blood pressure monitoring every 5 minutes.

2. If $\mathrm{SBP}$ is $>180 \mathrm{~mm} \mathrm{Hg}$ or MAP is $>130 \mathrm{~mm} \mathrm{Hg}$ and there is evidence of or suspicion of elevated ICP, then consider monitoring ICP and reducing blood pressure using intermittent or continuous intravenous medications to keep cerebral perfusion pressure $>60$ to $80 \mathrm{~mm} \mathrm{Hg}$.

3. If $\mathrm{SBP}$ is $>180 \mathrm{~mm} \mathrm{Hg}$ or MAP is $>130 \mathrm{~mm} \mathrm{Hg}$ and there is not evidence of or suspicion of elevated ICP, then consider a modest reduction of blood pressure (e.g., MAP of 110 $\mathrm{mm} \mathrm{Hg}$ or target blood pressure of $160 / 90 \mathrm{~mm} \mathrm{Hg}$ ) using intermittent or continuous intravenous medications to control blood pressure, and clinically reexamine the patient every 15 minutes.

SBP indicates systolic blood pressure; MAP, mean arterial pressure

Broderick J, Connolly S, Feldman E, Hanley D, Kase C, Krieger D, Mayberg M, Morgenstern L, Ogilvy CS, Vespa P, Zuccarello M. Guidelines for the Management of Spontaneous Intracerebral Hemorrhage in Adults. 2007 Update: A Guideline From the American Heart Association/American Stroke Association Stroke Council, High Blood Pressure Research Council, and the Quality of Care and Outcomes in Research Interdisciplinary Working Group. Stroke 1 June 2007; 38(6): 2001-2023.

Recent studies are clarifying the risk of ICH and ICH-related mortality associated with anticoagulation. Due to its predictable and reproducible anticoagulant effects, LMWH has become increasingly preferred over unfractionated heparin (UFH) for anticoagulation in the inpatient setting. LMWH and UFH have similar rates of major bleeding, ${ }^{5}$ yet studies comparing LMWH to UFH in patients with ST-elevation myocardial infractions who have also received thrombolytics have shown an increased risk of ICH in patients receiving LMWH over those receiving UFH. ${ }^{6}$ Predictors of anticoagulant-related ICH included coronary artery disease, atrial fibrillation, history of ischemic stroke, and history of pulmonary embolus or deep vein thrombosis. Patients with anticoagulant-related ICH also had a higher mortality rates than other ICH patients. The difference in mortality rates within the first 24 hours post event in one retrospective review was $33.2 \%$ in anticoagulant-related ICH versus $16.3 \%$ in patients with ICH not on anticoagulation. ${ }^{7}$ Anti-platelet therapy is also associated with worse clinical outcomes and is an independent predictor for acute hematoma enlargement, rapid death, and need for emergent hematoma evacuation. ${ }^{8}$

There is no proven method for reversing the effects of LMWH. Unfractionated heparin and LMWH exert their effects by binding to and catalyzing antithrombin III, which inhibits certain coagulation factors, particularly factor IIa and factor Xa. LMWH has a reduced ability to inhibit factor IIa compared to UFH, but has a similar effect on factor Xa. Intravenous protamine in animal studies and in vitro studies significantly neutralizes the factor IIa activity of LMWH, but only neutralizes $60 \%$ of its anti-factor Xa activity. Moreover, studies demonstrating or refuting protamine's beneficial effect on LMWH-related bleeding in humans are lacking. Nevertheless, in a patient who received LMWH within an 8 hour time window, the recommended approach is to administer $1 \mathrm{mg}$ of IV protamine for every 100 anti-factor Xa units of LMWH. One milligram of enoxaparin is equal to 100 anti-factor Xa units. Therefore, a patient who received $80 \mathrm{mg}$ of subcutaneous enoxaparin within 8 hours should be given 80 $\mathrm{mg}$ of IV protamine. A second dose of $0.5 \mathrm{mg}$ of IV protamine per 100 anti-factor Xa units may be given if bleeding continues. If LMWH was administered more than 8 hours ago, a lower initial dose of protamine is recommended. For UFH, $1 \mathrm{mg}$ of IV protamine will neutralize 100 units of UFH. ${ }^{5}$

\section{Conclusion}

In summary, we have presented a case of severe intracerebral hemorrhage in a patient receiving therapeutic enoxaparin and antiplatelet agents. Initial suspicion for acute intracranial hemorrhage was low based on non-localizing neurological exam and lack of head trauma, but remained within the immediate differential given the patient's history and current medications. The patient exhibited signs of slow global neurologic deterioration, but his exam remained non-focal throughout his hospital course. In this instance, radiographic evaluation was pursued promptly. Although the intent was to rule out, rather than confirm, suspected intracranial pathology, radiographic imaging remained an integral part of the complete patient evaluation.

Our experience suggests that while ICH classically presents with focal deficits, the absence of focal deficits does not exclude the diagnosis. It should remain high on any physician's differential while caring for a patient with an acute mental status change, particularly in a patient on therapeutic anticoagulation or antiplatelet medications. In managing patients with an $\mathrm{ICH}$, prompt neurosurgical evaluation is required. Additional immediate attention should focus on controlling hypertension, elevated intracranial pressure, hyperthermia, and hyperglycemia; reversing anticoagulants if needed; and monitoring the patient in an intensive care unit.

\section{References}

1. Broderick J, Connolly S, Feldman E, Hanley D, Kase C, Krieger D, Mayberg 
M, Morgenstern L, Ogilvy CS, Vespa P, Zuccarello M. Guidelines for the Management of Spontaneous Intracerebral Hemorrhage in Adults. 2007 Update: A Guideline From the American Heart Association/American Stroke Association Stroke Council, High Blood Pressure Research Council, and the Quality of Care and Outcomes in Research Interdisciplinary Working Group. Stroke 1 June 2007; 38(6): 2001-2023.

2. Leira R, Dávalos A, Silva Y, Gil-Peralta A, Tejada J, Garcia M, Castillo J; Stroke Project, Cerebrovascular Diseases Group of the Spanish Neurological Society. Early neurologic deterioration in intracerebral hemorrhage; Predictors and associated factors. NEUROLOGY 10 August 2004; 63(3): 461-467.

3. Poungvarin $\mathrm{N}$, Bhoopat $\mathrm{W}$, Viriavejakul A, Rodprasert $\mathrm{P}$, Buranasiri $\mathrm{P}$, Sukondhabhant S, Hensley MJ, Strom BL. Effects of dexamethasone in primary supratentorial intracerebral hemorrhage. NEJM, 14 May 1987; 316(20): 1229-1233.

4. Passer S, Rocchi R, Rossi S, Ulivelli M, Vatti G. Seizures after Spontaneous Supratentorial Intracerebral Hemorrhage. Epilepsia October 2002; 43 (10): $1175-1180$.
5. Hirsh J and Raschke R. Heparin and Low-Molecular-Weight Heparin The Seventh ACCP Conference on Antithrombotic and Thrombolytic Therapy. Chest September 2004; 126(3 supplement): 188S-203S.

6. Antman EM, Morrow DA, McCabe $\mathrm{CH}$, et al. Enoxaparin versus Unfractionated Heparin with Fibrinolysis for ST-Elevation Myocardial Infarction. New England Journal of Medicine 6 April 2006; 354(14): 1477-1488.

7. Flaherty ML, Haverbusch M, Padmini S, et al. Location and outcome of anticoagulant-associated intracerebral hemorrhage. Neurocritical Care December 2006; 5(3): 197-201.

8. Toyoda K, Okada Y, Minematsu K, et al. Antiplatelet therapy contributes to acute deterioration of intracerebral hemorrhage. Neurology 11 October 2005; 65(7): 1000-1004.Broderick J, Connolly S, Feldman E, Hanley D, Table 1. Suggested Recommended Guidelines for Treating Elevated Blood Pressure in Spontaneous ICH 